УДК 681.586 .5

\author{
О.В. Бурдышева ${ }^{1,2}$, И.Л. Никулин ${ }^{1}$, Е.С. Шолгин ${ }^{1,2}$ \\ ${ }^{1}$ Пермский национальный исследовательский политехнический университет, \\ Пермь, Россия \\ 2 Лаборатория агробиофотоники Пермского федерального \\ исследовательского центра Уральского отделения Российской академии наук, \\ Пермь, Россия
}

\title{
ЭКСПЕРИМЕНТАЛЬНАЯ РЕАЛИЗАЦИЯ
}

КВАЗИРАСПРЕДЕЛЕННОГО ВОЛОКОННО-ОПТИЧЕСКОГО

\section{ДАТЧИКА ВИБРАЦИИ НА ОСНОВЕ АМПЛИТУДНЫХ}

ДАТЧИКОВ

\begin{abstract}
На основе разработанного авторами чувствительного элемента реализован квазираспределенный волоконно-оптический датчик вибрации, предназначенный для определения наличия вибраций и их параметров в контролируемых точках. Представлены результаты измерений на экспериментальной схеме мультиплексирования, реализованной методом OTDR. Показаны принципиальная различимость датчиков и зависимость рефлектограммы от воздействия вибрации.

Ключевые слова: волоконно-оптический датчик вибрации, квазираспределенный датчик, амплитудная модуляция, мультиплексирование датчиков.

\author{
O.V. Burdysheva ${ }^{1,2}$, I.L. Nikulin ${ }^{1}$, E.S. Sholgin ${ }^{1,2}$ \\ ${ }^{1}$ Perm National Research Polytechnic University, Perm, Russian Federation \\ ${ }^{2}$ Agrobiophotonics Laboratory Perm Federal Research Center, \\ Ural Branch of the Russian Academy of Sciences, Perm, Russian Federation
}

\section{EXPERIMENTAL IMPLEMENTATION OF A QUASI-DISTRIBUTED FIBER OPTIC VIBRATION SENSOR ON THE BASIS OF AMPLITUDE SENSORS}

\footnotetext{
On the basis of the sensitive element developed by the authors, a quasi-distributed fiber-optic vibration sensor has been implemented, designed to determine the presence of vibrations and their parameters at controlled points. The results of measurements on an experimental multiplexing scheme implemented by the OTDR method are presented. The principal distinguishability of the sensors and the dependence of the trace on the effects of vibration are shown.

Keywords: fiber-optic sensor vibration, quasi-distributed sensor, amplitude modulation, multiplexing sensors.
} 


\section{Введение}

Волоконно-оптические датчики (ВОД) в основном состоят из диэлектриков, не требуют электрического питания и заземления [1], следовательно, могут найти свое применение в различных отраслях. Они обладают хорошими показателями точности и производительности, могут обладать малым размером (до $0,1 \mathrm{~cm}^{2}$ для брэгговского чувствительного элемента) [2], имеют низкую стоимость и могут быть значительно удалены от регистрирующего оборудования. ВОД на разных принципах работы позволяют измерять большое количество параметров: давление, температуру, расстояние, вибрацию и т.д.

Среди разновидностей ВОД находятся амплитудные датчики. В результате физического воздействия на чувствительный элемент амплитудного волоконно-оптического датчика модулируется интенсивность распространяющегося по оптическому волокну светового сигнала. Амплитудные датчики имеют свои преимущества, они являются наиболее простыми и удобными в эксплуатации конструкциями. Действительно, большинство амплитудных ВОД не требует использования когерентных источников света, а их измерительные схемы не предъявляют специальных требований ни к источникам, ни к приемникам излучения, а также не нуждаются в дополнительной обработке выходных сигналов датчиков. На сегодняшний день разработано множество различных конструкций амплитудных ВОД, все их можно разделить на две основные группы [3].

К первой группе относятся амплитудные ВОД, в которых ОВ используется для передачи сигнала от источника к чувствительному элементу и от него к приемнику. Чувствительным элементом в таких датчиках являются конструкции из материалов, чувствительных к внешним физическим воздействиям, способные изменять интенсивность проходящего излучения [4]. Среди главных преимуществ - простота конструкций и высокая чувствительность датчиков, однако у датчиков такого типа есть существенные недостатки, связанные с необходимостью разрыва непрерывной волоконной линии для обеспечения ввода излучения в чувствительный элемент датчика, что приводит к значительным потерям световой мощности на элементах межсоединений. Кроме того, использование разнородных оптических компонентов обусловливает низкую механическую стабильность характеристик измерительных устройств. Данные недостатки 
не позволяют использовать их в распределенных измерительных системах.

В амплитудных ВОД второй группы волоконный световод используется в качестве чувствительного элемента. В таких датчиках для измерения величины внешнего воздействия используются явления, приводящие к эффекту модуляции интенсивности распространяющегося по световодам излучения. К этим явлениям относятся: зависимость эффективности процесса рэлеевского рассеяния излучения в световодах от внешних условий; нарушение явления полного внутреннего отражения и направленная связь мод, вызываемые внешним воздействием. ВОД данной группы построены на непрерывной волоконной линии, что позволяет мультиплексировать ВОД в квазираспределенную измерительную линию с использованием известного в волоконнооптических системах связи метода импульсно-временной рефлектометрии [5, 6, 7].

Квазираспределенные датчики состоят из множества точечных сенсорных элементов в одном общем световоде. Состояние каждого сенсорного элемента можно проанализировать независимо от других, так как они обладают каждый своим уникальным свойством, точность элементов определяет точность системы.

Распределенные и квазираспределенные волоконно-оптические датчики находят свое применение в области обеспечения безопасности, при эксплуатации технических объектов, мониторинге протяженных объектов, строительных конструкций, охране периметра. Мониторинг опасного уровня вибрации позволит оценить уровень износа конструкции и предотвратить ее разрушение.

Целью настоящей работы является реализация квазираспределенного волоконно-оптического датчика, предназначенного для определения наличия вибраций и их параметров в контролируемых точках.

\section{Чувствительный элемент}

Схема чувствительного элемента представлена на рис. 1, более подробно датчик описан в работах [8, 9]. Чувствительный элемент мультиплексируемых точечных амплитудных ВОД представляет собой участок волоконного световода, выполненный в виде петли 4, закрепленной на основании корпуса датчика 2, которое прикрепляется к контролируемой поверхности, на петле ОВ закреплен груз 3. Вдоль чувст- 
вительного волокна установлены бортики 5 для гашения горизонтальных колебаний.

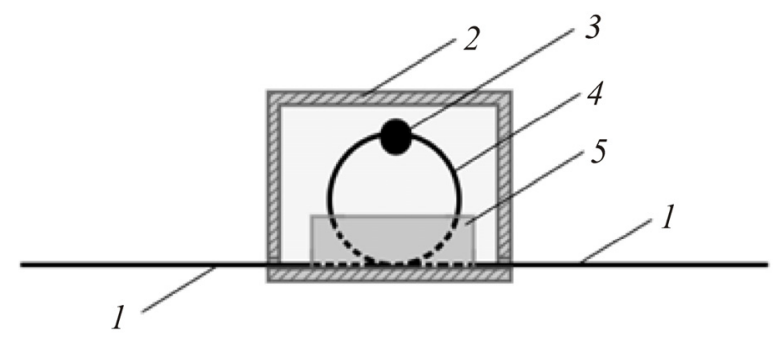

Рис. 1. Конструкция чувствительного элемента: 1 - оптическое волокно, подводящее/отводящее излучение, 2 - корпус датчика, 3 - груз, 4 - изогнутое волокно, 5 - бортики

Устройство работает следующим образом. Излучение от источника направляется к чувствительному элементу по волокну 1 , устойчивому к изгибам. Наличие вибрации вызывает вертикальные колебания груза, что, в свою очередь, приводит к изменению диаметра изогнутого волокна, часть излучения, проходящего через изогнутое волокно 4 , покидает световод по причине нарушения полного внутреннего отражения, следовательно, увеличиваются потери мощности.

В конструкции чувствительного элемента радиус изгиба оптического волокна составлял 18 мм, масса груза 70 мг, при заявленных параметрах оценка чувствительности датчика представлена в работе [8]. При амплитуде вибрации $A \approx 1$ мм и частоте $f=80$ Гц виброускорение $a_{\mathrm{B}}=A(2 \pi f)^{2}=25 g$, где $g-$ ускорение свободного падения. Изменение $\Delta P_{0,1}$ при амплитуде 0,1 мм соответствует виброускорению $2,5 \mathrm{~g}$, при 0,3 мм $-7,5 \mathrm{~g}$. Чувствительность амплитудного датчика в этом случае: $\left(\Delta P_{0,3}-\Delta P_{0,1}\right) / \Delta a_{\mathrm{B}}=0,18 \mathrm{MBT} / \mathrm{g}$.

\section{Реализация квазираспределенного датчика вибрации}

Мультиплексирование точечных амплитудных датчиков вибрации проведено на портативной измерительной платформе FTB-500 [10] при помощи рефлектометра FTB-7200D. Экспериментальная установка содержит рефлектометр FTB-7200D, две катушки SMF-28ultra ( 700 m и $\sim 500 \mathrm{~m}$ ), два амплитудных ВОД. Между ними вваривается дополнительное волокно 10 м, используемое для разделения датчиков. Экспериментальная установка представлена на рис. 2. 


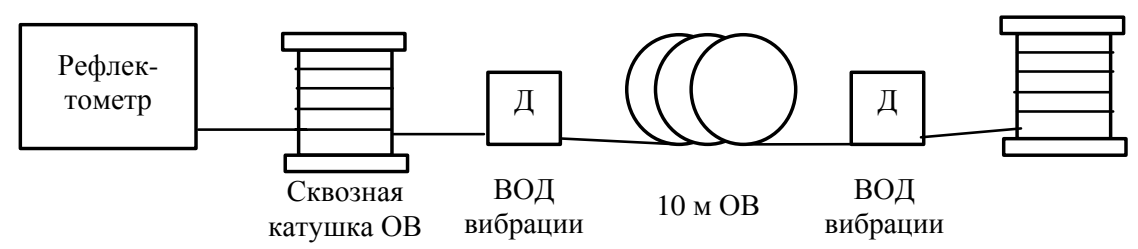

$a$

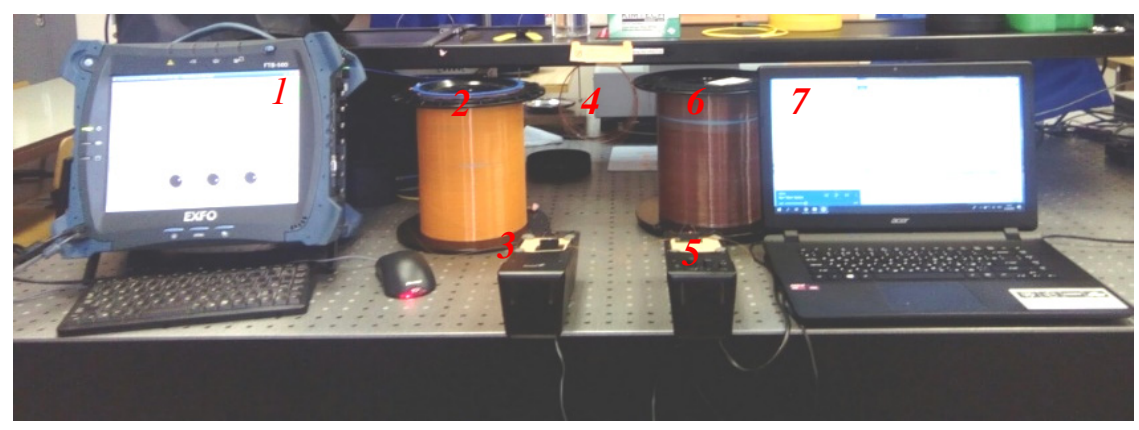

$\sigma$

Рис. 2. Экспериментальная установка: $a$ - схема, $\sigma$ - фото: 1 - рефлектометр (FTB-500 с модулем FTB-7200D), 2 - сквозная катушка № 1, 3 - датчик № 1, установленный на колонку, 4 - дополнительное волокно длиной 10 м, 5 - датчик № 2, установленный на колонку, 6 - катушка № 2 для удаления отражающего конца волокна, 7 - ПК, управляющий колонками

Установленные параметры работы рефлектометра: время усреднения $t=30 \mathrm{c}$, рабочая длина волны $\lambda=1550 \mathrm{Hм}$, длина импульса $P=3$ м, дальность замера $L=2,5$ км.

Результаты измерений представлены на рис. 3-6. Режимы подачи вибраций на датчики и соответствующие им графики приведены в таблице.

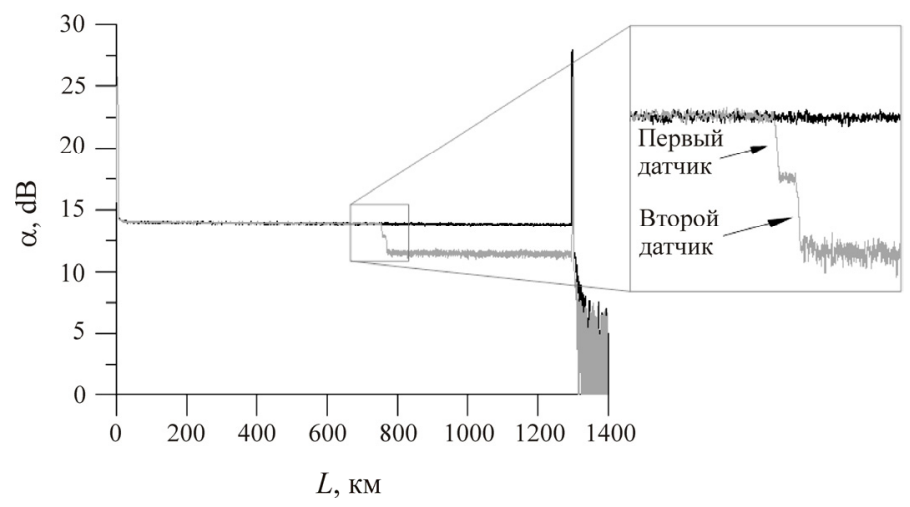

Рис. 3. Сравнение рефлектограмм с внесенными в схему датчиками и без них 


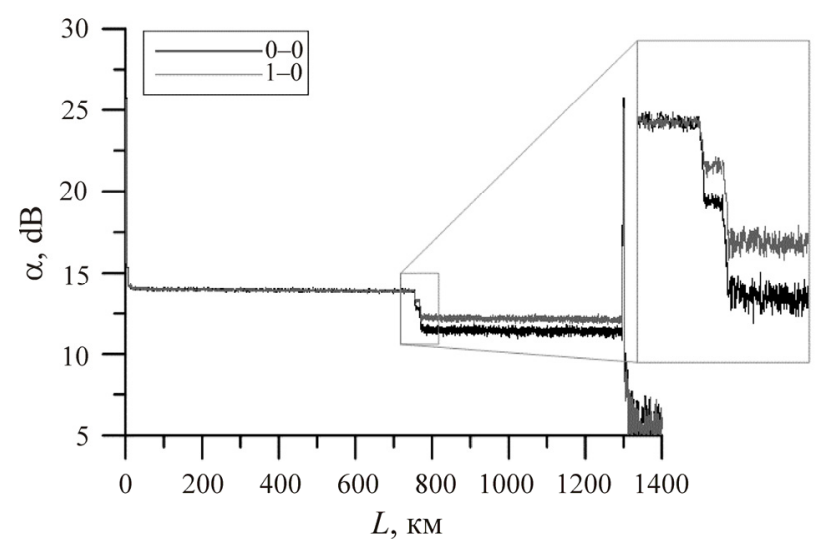

Рис. 4. Сопоставление рефлектограмм покоя и воздействия вибрации на первый датчик

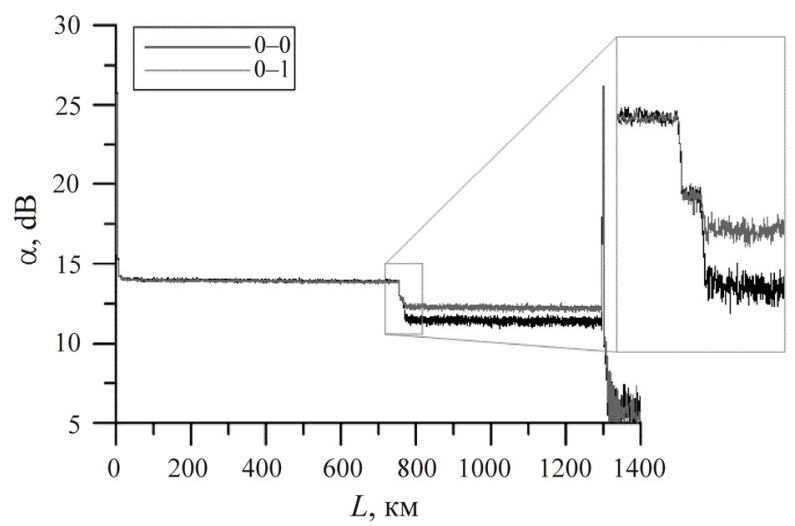

Рис. 5. Сопоставление рефлектограмм покоя и воздействия вибрации на второй датчик

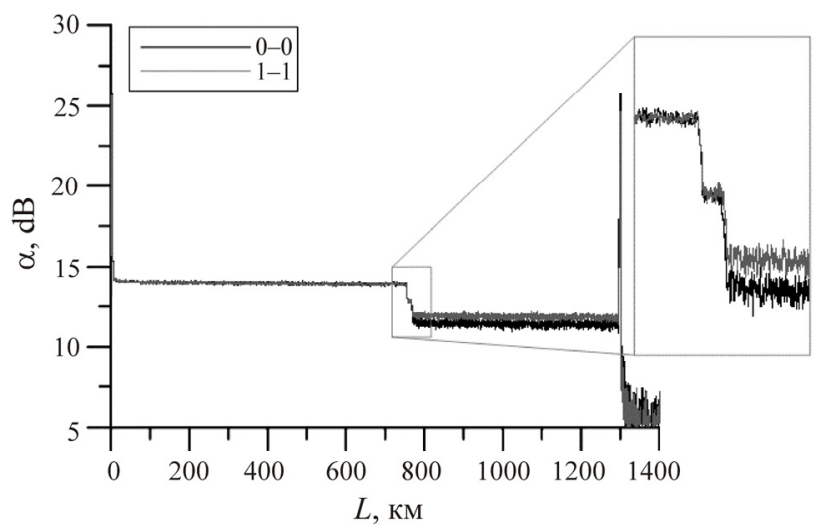

Рис. 6. Сопоставление рефлектограмм покоя и воздействия вибрации на оба датчика 
Режимы подачи вибраций на датчики

\begin{tabular}{|l|c|c|c|c|}
\hline \multicolumn{1}{|c|}{ Наименование } & \multicolumn{4}{c|}{ Режимы } \\
\hline Датчик № 1 & выкл. & вкл. & выкл. & вкл. \\
\hline Датчик № 2 & выкл. & выкл. & вкл. & вкл. \\
\hline Обозначение режима & $0-0$ & $1-0$ & $0-1$ & $1-1$ \\
\hline Рисунок & 3 & 4 & 5 & 6 \\
\hline
\end{tabular}

На рис. 3 представлено сравнение рефлектограмм, снятых с экспериментальной схемы и с волокна без датчиков, видно, что потери на сварке не существенны по сравнению с потерями на датчиках, а именно на изгибе волокна. На рефлектограмме режима без воздействия вибрации «0-0» видны различимые потери мощности (см. рис. 3), которые соответствуют двум датчикам. На рис. 4 показана рефлектограмма режима «1-0». Видно изменение величины ступени, соответствующей потерям мощности на первом датчике, на который действует вибрация. Наблюдается уменьшение потерь относительно начального сигнала «0-0». На рис. 5 (режим «0-1») изменяется величина ступени второго датчика, так же, как и в первом случае, уменьшаются потери относительно начального сигнала «0-0». При воздействии вибрации на оба датчика (режим «1-1») потери уменьшаются относительно начального сигнала $\ll 0-0 »$ на обоих (рис. 6).

\section{Заключение}

Реализована экспериментальная схема, позволяющая регистрировать вибрации в контролируемых точках методом OTDR на амплитудных датчиках вибрации. Экспериментально показана различимость на рефлектограмме двух датчиков вибрации. Продемонстрирована возможность по уровню мощности отраженного излучения определять наличие или отсутствие вибраций на чувствительном элементе. Таким образом, показана принципиальная возможность мультиплексирования амплитудных волоконно-оптических датчиков вибрации методом OTDR.

\section{Список литературы}

1. Гончаренко И.А., Рябцев В.Н. Датчики контроля состояния инженерных и строительных конструкций на основе оптических волноводных структур // Вестник Командно-инженерного института МЧС Республики Беларусь. - 2013. - № 2(18). - С. 118-132. 
2. Возможности, задачи и перспективы волоконно-оптических измерительных систем в современном приборостроении / В.Б. Гармаш, Ф.А. Егоров, Л.Н. Коломиец, А.П. Неугодников, В.И. Поспелов // Спецвыпуск «Фотон-экспресс». Наука. - 2005. - № 6. - С. 128-140.

3. Бусурин В.И., Носов Ю.Р. Волоконно-оптические датчики: Физические основы, вопросы расчета и применения. - М.: Энергоатомиздат, 1990. - $256 \mathrm{c}$.

4. Кузнецов В.П., Иванов А.А., Кудряшов Б.П. Проектирование средств измерения параметров технологических объектов на основе волоконно-оптических преобразователей: учеб. пособие. - Курган: Изд-во Курган. гос. ун-та, 2013. - 84 с.

5. Листвин А.В., Листвин В.Н. Рефлектометрия оптических волокон. - М.: ЛЕСАРарт, 2005. - 208 с.

6. Кульчин Ю.Н. Распределенные волоконно-оптические датчики и измерительные сети. - Владивосток: Дальнаука, 1999. - 283 с.

7. Оптический рефлектометр / В.Г. Воронин, О.Е. Наний, А.А. Кулик, А.Н. Туркин. - М., 2007. - 18 с.

8. Бурдышева О.В., Никулин И.Л. Амплитудный волоконнооптический датчик вибрации // Фотоника. - 2019. - № 1. - С. 80-85.

9. Никулин И.Л., Бурдышева О.В. Волоконно-оптическое устройство регистрации вибраций: пат. № 179547 Рос. Федерация. МПК G01P 15/02 (2013.01); заяв. 2017139623, 14.11.2017; опубл. 17.05.2018. Бюл. № 14.

10. Оборудование, приборы, инструменты для работы на ВОЛС (для работы с ВОК). Приборы для обслуживания волоконной оптики EXFO [Электронный pecypc]. - URL: https://www.exfo.com (дата обращения: 15.05.2019).

\section{References}

1. Goncharenko I.A., Riabtsev V.N. Datchiki kontrolia sostoianiia inzhenernykh i stroitelnykh konstruktsii na osnove opticheskikh volnovodnykh struktur [Sensors for monitoring the state of engineering and building constructions based on optical waveguide structures]. Vestnik Komandnoinzhenerskogo institutata MChS Respubliki Belarus, 2013, no. 2 (18), pp. 118-132.

2. Garmash V.B., Egorov F.A., Kolomiets L.N., Neugodnikov A.P., Pospelov V.I. Vozmozhnosti, zadachi i perspektivy volokonno-opticheskikh 
izmeritelnykh sistem $\mathrm{v}$ sovremennom priborostroenii [Opportunities, problems and perspectives in fiber-optic measuring systems in modern instrumentation]. Spetsvypusk "Foton-ekspress" - Nauka, 2005, no. 6, pp. 128-140.

3. Busurin V.I., Nosov Iu.R. Volokonno-opticheskie datchiki: fizicheskie osnovy, voprosy rascheta i primeneniia [Fiber-optic sensors: physical basics, calculation and application issues]. Moscow, Energoatomizdat, 1990, $256 \mathrm{p}$.

4. Kuznetsov V.P., Ivanov A.A., Kudriashov B.P. Proektirovanie sredstv izmereniia parametrov tekhnologicheskikh obektov na osnove volokonno-opticheskikh preobrazovatelei [Measuring instruments design for the parameters of technological objects based on fiber-optic converters]. Kurgan, Izdatelstvo kurganskogo gosudarstvennogo universiteta, 2013, 84 p.

5. Listvin A.V., Listvin V.N. Reflektometriia opticheskikh volokon [Optical fiber reflectometry]. Moscow, LESARart, 2005, 208 p.

6. Kulchin Iu.N. Raspredelennye volokonno-opticheskie datchiki i izmeritelnye seti [Distributed fiber-optic sensors and measurement networks]. Vladivostok, Dalnauka,1999, 283 p.

7. Voronin V.G., Nanii O.E., Kulik A.A., Turkin A.N. Opticheskii reflektometr [Optical reflectometer]. Moscow, 2007, 18 p.

8. Burdysheva O.V., Nikulin I.L. Amplitudnyi volokonnoopticheskii datchik vibratsii [Amplitude fiber-optic vibration sensor]. Photonics, 2019, no. 1, pp. 80-85.

9. Nikulin I.L., Burdysheva O.V. Volokonno-opticheskoe ustroistvo registratsii vibratsii [Fiber-optic vibration detector]. Patent na poleznuiu model no. 179547 Russian Federation (2018).

10. Oborudovanie, pribory, instrumenty dlia raboty na VOLS (dlia raboty s VOK). Pribory dlia obsluzhivaniia volokonnoi optiki exfo [Equipment, instruments, tools for working at fiber-optic lines (for working with FOCL). Fiber-optic service EXFO sevices], available at: https://www.exfo.com (accessed 15 May 2019).

Получено 17.06.19 